\title{
Dictionaries merger for text expansion in question answering
}

\author{
Bernard JACQUEMIN \\ b_jacquemin@yahoo.fr
}

\begin{abstract}
This paper presents an original way to add new data in a reference dictionary from several other lexical resources, without loosing any consistence. This operation is carried in order to get lexical information classified by the sense of the entry. This classification makes it possible to enrich utterances (in QA: the queries) following the meaning, and to reduce noise. An analysis of the experienced problems shows the interest of this method, and insists on the points that have to be tackled.
\end{abstract}

\section{Introduction}

Our society is currently facing an increasing amount of textual data, that no-one can store up or even read. Many automatic systems are designed to find a requested piece of information. All the current systems use dictionaries to identify data in texts or in queries. QA softwares, which are particularly demanding about data from the dictionary, have a similar mode of working: they process an utterance (generally the query) in order to provide the largest number of way to express the same meaning. Then they try to find a match between the expanded utterance and a text. For example, (Hull, 1999) expands synonymically the 'significant' vocabulary of the question. QUALC (Ferret et al., 1999) adds stemming expansion prior to using a search engine. The Falcon system (Moldovan et al., 2000) uses some semantic relations from WordNet when it expands the question.

In this paper, I present a way to process dictionaries to make them consistent with the needs of the application. I first describe the lexical needs of my QA application. I secondly outline the issue of the use of several incompatible dictionaries. Then I show the way I distribute information from additional dictionaries to a reference one: synonyms, derivative forms and taxonomy. Finally, I present the problems and difficulties I found.

\section{What the QA method needs}

The QA system (Jacquemin, 2003) is based on a matching procedure between query and text segment. As most of the other approaches, my methodology solves the problem of the different ways to express the same idea by adding to the utterance ('enrichments') synonyms, derivatives or words belonging to the same taxonomy.

My method entails two new features: First, it uses semantic disambiguation in order to choose the right meaning to each word in the sentences. I notice that most of the QA systems try to give as many enrichments as possible to a word rather than to a meaning. The answers often correspond to a sense different from the original one. But if each enrichment has the same sense as the original one, the noise decreases.

The fact that a semantic disambiguator needs a large context to the word to be disambiguated (Weaver, 1949) provides the second feature: the query generally comprises few words. I decided to process the documents to build an enriched informative structure (Jacquemin, 2004). But this feature falls outside the scope of this paper.

My semantic disambiguator (Jacquemin et al., 2002) is an evolution of a tool previously developed for both French and English at XRCE (Brun, 2000; Brun et al., 2001). The idea is to use a dictionary as a tagged corpus to extract semantic disambiguation rules. The contextual data (syntactic, lexico-syntactic and semanticosyntactic) for a given sense of a word are seen as differential indications. So when the schema is found in the context of this word in a sentence, the corresponding sense is assigned.

In figure 1 , we can see how a disambiguation rule is extracted from an informative field of Dubois' French dictionary (Dubois and DuboisCharlier, 1997). From the instance field of the entry remporter in its second sense gagner (to win), the XIP parser (Aït-Mokhtar et al., 2002) extracts a lexico-syntactic schema: VARG [DIR] 
Example from Dubois' dictionary (entry: remporter):

On remporte la victoire sur ses adversaires (sense nb 2 : gagner)

We win a victory over our adversaries.

\section{Dependency containing remporter:}

VARG [DIR] (remporter, victoire)

Corresponding disambiguation rule: remporter: VARG [DIR] (remporter, victoire) $==>$ sense gagner

Figure 1: Extraction of a semantic disambiguation rule.

means that the argument victoire is a direct object of the argument remporter. The rule built from this dependency indicates that the sense of the word remporter, in a context where victoire (victory) is the direct object, is the second sense gagner (to win). Two other types of rules exists: the first type puts lexical rules into general use, replacing lexical arguments by corresponding semantic classes. The other one uses syntactic schemas stipulated by the dictionary (for instance: transitive, reflexive, etc.).

The dictionary needs of both QA system and semantic disambiguator are of two natures. First, the dictionary is required to share out data following sense and not following lemma: The data are differential indications. Second, the dictionary is required to contain contextual information as much as possible: examples or collocations (lexical rules), semantic classes or application domains (generalized rules), subcategorisation... The Dubois' dictionary yields to these demands, and moreover it contains some data that could be helpful to enrich an utterance: synonyms, instructions for derivations...

\section{Enrichment problems}

Several expansion solutions are proposed by the QA approaches: use of synonyms or taxonomy's members, stemming or use of derivatives...

Dubois' dictionary contains some synonyms linked with a sense of the word they are synonymous with. But these synonyms are too few to provide sufficient enrichments. The system needs one or more synonyms dictionaries to complete Dubois' gaps. No synonyms dictionary shares out the synonyms by sense of the entry, except Euro WordNet (Catherin, 1999). But Euro WordNet's sharing out into senses does not match Dubois' senses. Thus the question is to distribute the available synonyms of each word to the right sense in Dubois'.

The stemming, which considers two words with the same stem nearly synonyms, is too unpredictable to be used in a methodology that tries to avoid noise. As Dubois' provides instructions to form derivatives from lemma and suffixes for some senses, the derivation is preferred to the stemming. But the instructions are often vague, and indicate only the suffix to use and the new part-of-speech. It is not sufficient to be used automatically. Thus the derivation procedure needs an extra tool able to propose derivatives, including the right one. Dubois' information is sufficient to filter and classify them.

Finally, Dubois' does not provide taxonomy, and the French resources containing a semantic hierarchy do not supply contextual information. The taxonomy has to be found in another resource, which is not consistent with the reference dictionary. The compatibility between senses of all these resources is the objective.

\section{How to make the dictionaries compatible}

The main difficulty is to share out information collected from extra dictionaries. The dictionaries are incompatible with Dubois', but new data have to be distributed following the senses of the entries of the reference dictionary.

\subsection{Synonyms}

Three resources are at my disposal: Bailly's dictionary (Bailly, 1947), an electronic dictionary designed by Memodata, and the French Euro WordNet (Catherin, 1999). The expansion methods commonly use all the available synonyms for a word, but my approach has to keep only the synonyms corresponding to the current sense of the word. For each considered sense for a word, Dubois' provides semantic features: a semantic class and an application domain.

The synonyms from the extra dictionaries are proposals. A proposal for a lemma in Dubois' dictionary is kept for a given sense only if one sense at least of the Dubois' entry corresponding to the proposal matches the semantic features of the given sense. If no sense of the proposal matches the semantic features of the given sense, the proposal is rejected for this sense.

In figure 2, the problem is to determine which proposal matches the word ravir in the sense nb 2 voler (to steal). The semantic features of this 
Semantic features of the entry:

Domain Class

ravir (2) SOC $\quad \mathrm{S} 4$

\begin{tabular}{lll}
\multicolumn{3}{c}{ Semantic features of synonyms: } \\
& Domain & Class \\
charmer & PSY & P2 \\
voler & SOC & S4
\end{tabular}

Figure 2: Selection of the synonyms.

sense are the application domain SOC (sociology) and the semantic class S4 (to grip, to own). The proposal charmer, which features are PSY (psychology) and P2 (psychological verb) does not match the features of ravir 2 . The proposal dérober in its second and fourth senses has the same features. This proposal is confirmed for ravir in sense nb 2 . It will be used as enrichment when the sense nb 2 of ravir is detected in an utterance by the semantic disambiguator. This procedure is applied for all the proposed synonyms for all the senses of each entry in Dubois'.

\subsection{Derivatives}

The derivation field in the Dubois' provides sufficient indications to recognize the stipulated derivatives of an entry in a determined sense. Thus, the need is a resource or a tool providing all the potential derivative from a word. Resources are rare and incomplete for French, but I have to my disposal a tool (Gaussier et al., 2000) able to construct suffixal derivatives from a word. If the only constraint requires the derivatives belong to the lexicon, all the right suffixal forms are provided among the incorrect proposals. When all the proposals are produced, the suffix of each proposal is compared with the instructions in the dictionary. When they match, the derivative is kept for the current sense. If not, the derivative is rejected.

\begin{tabular}{l|c|l} 
Derivatives for the verb couper: \\
$\begin{array}{l}\text { Proposed } \\
\text { derivatives }\end{array}$ & $\begin{array}{c}\text { Instruction } \\
\text { sense nb 1 }\end{array}$ & $\begin{array}{l}\text { Retained } \\
\text { derivatives }\end{array}$ \\
\hline coupure & ure & coupure \\
coupable & - & removed \\
coupage & age sense 5$)$ & removed \\
coupeur & eur & coupeur \\
coupant & ant & coupant \\
$\ldots$ & $\ldots$ & $\ldots$
\end{tabular}

Figure 3: Selection of the derivatives.

The figure 3 shows the working of the method.
For the verb couper in the sense trancher (to cut, to slice), Dubois' indicates derivatives with suffixes -ure: coupure (break), -ant: coupant (sharp) and -eur: coupeur (cutter). But no instruction is given for a suffix -able. The wrong derivative coupable (guilty) is rejected.

\subsection{Taxonomy}

Only two resources containing taxonomy exist for French. AlethDic (Gsi, 1993) is known for its very bad quality. The hierarchy is neither very deep, nor very large. The semantic relations are not strictly defined inside the hierarchy. Because of this, I rejected AlethDic.

The other resource is EuroWordNet. Two kind of taxonomic relations are defined: hyperonymy (and hyponymy), and meronymy (and holonymy). The other semantic relations of this resource fall outside the scope of this paper.

The taxonomic relations link synsets together. The synsets contain synonymous words for at least one of their senses. The taxonomy is usable by the QA system only if the sense of the whole synset can be identified, and if the sense matches at least one of the sense of the word under consideration in Dubois' dictionary.

So each word in Dubois' has to be linked with a synset to be inserted into a taxonomic hierarchy. That amounts to match senses in Dubois' and synsets in EuroWordNet. We already have some senses in Dubois' matching sets of synonyms in EuroWordNet. It is easy to use the additional synonyms from Euro WordNet to set up a correspondence between sense of Dubois' dictionary and synsets of EuroWordNet.

The procedure is to examine all the synsets where a considered word appears. For each of its sense, if the majority of the synonyms obtained from EuroWordNet are contained in a synset, the meaning illustrated by the synset and this sense of the word are considered to be equivalent. In this case, the word under consideration is inserted in this place into the taxonomic hierarchy. Otherwise, the synset is not seen to match the sense, and it is rejected.

\section{Experienced problems}

The difficulties met differ for each kind of processed data. In the sharing out of the synonyms, the system cannot determine automatically the meaning of a multiword expression. Dubois' only lists single words, and no semantic feature can be allocated to a multiword expression. A multiword proposal is considered to have all the meaning of the word to which it is synonym. 
I have no real evaluation of this procedure: the division into senses of the reference dictionary is as always open to doubt. Considering the result, examiners never agree with each synonyms for a sense. But when they agree (three examiners where consulted), they where satisfied by more than $80 \%$ of the synonyms.

The derivation tool provides nearly all the derivatives from a word when no constraint is defined. Most of the wrong derivatives (about $97 \%$ ) are screened by the instructions supplied by Dubois' dictionary. However, these figure are not valid for short words: the tool is designated in such a way that derivatives with a radical shorter than 3 letters are generally wrong. Moreover, the instructions are often incomplete in the dictionary, above all nominal entries.

The promising procedure using taxonomy, presented above, is still a suggestion. I am facing with the problem that EuroWordNet covers only a small part of the French lexicon. A more proper trial should use WordNet (Fellbaum, 1998), that covers a huge part of the English lexicon, in an English QA application.

\section{Conclusion}

In this paper, I present an original method to merge several dictionaries in such a way that all the informative fields become semantically consistent. This need comes from an expansion method for QA, which uses as enrichment only the synonyms, derivatives, and taxonomy that match the sense determined by a semantic disambiguator. The method to share out information is particular for each kind of enrichment. An analysis of the experienced problems shows the interest of the method, and insists on the points that still have to be tackled.

In a more general perspective, it is known that no perfect dictionary exists. Each dictionary used by this method has gaps. The method used to mix information is filtering data from extra dictionaries by data from a reference dictionary, but errors in the reference are passed on to the added data. The right solution should be to use more than one reference dictionary.

\section{References}

Salah Aït-Mokhtar, Jean-Pierre Chanod, and Claude Roux. 2002. Robustness beyond shallowness: incremental deep parsing. Natural Language Engineering, 8(2/3):121-144.

René Bailly. 1947. Dictionnaire des synonymes de la langue française. Larousse, Paris.
Caroline Brun, Bernard Jacquemin, and Frédérique Segond. 2001. Exploitation de dictionnaires électroniques pour la désambiguïsation sémantique lexicale. $T A L$, 42(3):667-690.

Caroline Brun. 2000. A client/server architecture for word sense disambiguation. In Proceedings of Coling'2000, pages 132-138, Saarbrücken, Deutschland.

Laurent Catherin. 1999. The french wordnet. Technical report, EuroWordNet.

Jean Dubois and Françoise Dubois-Charlier. 1997. Dictionnaire des verbes français. Larousse, Paris. Electronic version, with its complement: Dictionnaire des mots.

Christiane Fellbaum, editor. 1998. WordNet: an electronic lexical database. Language, Speech and Communication. The MIT Press, Cambridge, Massachusetts.

Olivier Ferret, Brigitte Grau, Gabriel Illouz, Christian Jacquemin, and N. Masson. 1999. Qualc. The question-answering program of the langage et cognition group at limsi-cnrs. In Proceedings of TREC-8, pages 455-464.

Éric Gaussier, Gregory Grefenstette, David Hull, and Claude Roux. 2000. Recherche d'information en français et traitement automatique des langues. TAL, 41(2):473-493.

Gsi-Erli, France, 1993. Le dictionnaire AlethDic, 1.5 edition, Mars.

David A. Hull. 1999. Xerox trec-8 question answering track report. In Proceedings of TREC-8, pages 743-752.

Bernard Jacquemin, Caroline Brun, and Claude Roux. 2002. Enriching a text by semantic disambiguation for information extraction. In LREC 2002 Workshop Proceedings.

Bernard Jacquemin. 2003. Construction et interrogation de la structure informationnelle d'une base documentaire en français. Ph.D. thesis, Université de la Sorbonne Nouvelle.

Bernard Jacquemin. 2004. Analyse et expansion des textes en question-réponse. In Actes des Yèmes JADT, volume 2, pages 633-641.

Dan Moldovan, Sanda Harabagiu, Marius Psca, Rada Mihalcea, Richard Goodrum, Roxana Grju, and Vasile Rus. 2000. The structure and performance of an open-domain question answering system. In Proceedings of the 38th Annual Meeting of the ACL, pages 563-570.

Warren Weaver, 1949. Machine translation of languages, chapter Translation, pages 15-23. John Wiley and Sons, New York. 